\title{
Prompt photon production in high-energy $p A$ collisions at forward rapidity
}

\author{
G. Sampaio dos Santos, ${ }^{1}$ G. Gil da Silveira $\odot,{ }^{1,2, *}$ and M. V. T. Machado ${ }^{1}$ \\ ${ }^{1}$ High Energy Physics Phenomenology Group, GFPAE IF-UFRGS, Caixa Postal 15051, CEP 91501-970, Porto Alegre, RS, Brazil \\ ${ }^{2}$ Departamento de Física Nuclear e de Altas Energias, Universidade do Estado do Rio de Janeiro, CEP 20550-013, Rio de Janeiro, RJ, Brazil
}

(Received 21 June 2020; accepted 9 October 2020; published 2 November 2020)

\begin{abstract}
Prompt photon production in hadronic collisions at energies available at RHIC and at the LHC is investigated within the QCD color dipole approach. Predictions for the nuclear modification factor in $p A$ collisions are evaluated based on the parton saturation framework, and the results are compared with the experimental measurements as a function of the photon transverse momentum at different rapidity bins. The reliability of the models is performed with the data from the PHENIX, ATLAS, and ALICE Collaborations. Moreover, we show that the observed $x_{T}$ scaling of prompt photon production in $p p$ and $p A$ collisions can positively be addressed in the QCD color dipole formalism.
\end{abstract}

DOI: 10.1103/PhysRevC.102.054901

\section{INTRODUCTION}

In high-energy collisions involving a nuclei, the presence of effects associated with the nuclear environment modify the behavior of the partonic distributions. A detailed understanding of the initial- and final-state effects associated with the stages of the collision is crucial to describe the data from heavy-ion collisions (HICs) at the BNL Relativistic Heavy Ion Collider (RHIC) and the Large Hadron Collider (LHC). The suitable scenario to analyze such effects would be the nuclear deep-inelastic scattering (nDIS), which is the plan of the future Electron-Ion Collider [1]. Alternatively, proton-nucleus $(p A)$ collisions can be used as a probe of the nuclear effects, since the formation of a quark-gluon deconfined medium known as Quark-Gluon Plasma (QGP) is not expected in this case. For a better understanding of the scenario created in $A A$ reactions, $p A$ collisions can be used as a baseline to disentangle the initial- and final-state effects. Hence, one needs to evaluate such effects before testing the signals from the highdensity QCD medium that can be identified in $A A$ collisions. Consequently, a consistent knowledge of the measurements in $p A$ collisions is essential to improve the comprehension of the underlying physics in HICs. Usually, analyzing the nuclear effects is made by measuring a nuclear modification factor, which can establish a reference for the collision centrality or system-size dependence. At RHIC energy [2-4], a suppression was observed for pion production in $d \mathrm{Au}$ collisions, and such a particular result is an important source to constrain the nuclear parton distribution function (nPDF). At the LHC, investigations about the nuclear modification factor for $\pi^{0}$

*Corresponding author: gustavo.silveira@ cern.ch

Published by the American Physical Society under the terms of the Creative Commons Attribution 4.0 International license. Further distribution of this work must maintain attribution to the author(s) and the published article's title, journal citation, and DOI. Funded by $S C O A P^{3}$. and the ratio of prompt photons to pion production, $\gamma / \pi^{0}$, have been used to verify the self-consistency of the QCD approaches (see, for instance, discussions in Refs. [5-7]).

Here we focus on an important hard probe of the nuclear environment, namely, the production of hard isolated photons. At the high-energy regime, the nucleus target is probed at small Bjorken variable $x$, and such a kinematic region can be accessed on measurements of prompt photons at forward rapidities. Measurements of prompt photon cross sections have been proposed as a clean source of information about the QCD dynamics [8-11]. Due to the nature of the quark-photon vertex, the only interaction is electromagnetic, especially because photons are colorless probes of the dynamics of quarks and gluons. Also, direct photons are not disturbed by final interactions, then they can leave the system without loss of energy and momentum. Another useful property is the elementary diagrams for the underlying processes, which are theoretically well established and the contribution from fragmentation processes can be suppressed by an isolation criteria. Studies of nPDFs using prompt photons have been proposed in Ref. [12], demonstrating that experimental data on this process can strongly constrain them. In particular, gluon distribution, which are not well constrained at small $x$ and there are large theoretical uncertainties from the usual perturbative QCD (pQCD), can be extracted in a precise way. Toward low values of $x$, the gluon density substantially increases, bringing concerns about unitarity violation. At the low- $x$ regime the growth of the gluon density can be controlled by the gluon recombination effect, which is a nonlinear QCD phenomenon leading to gluon saturation; it is expected that the low- $p_{T}$ photon distribution can probe this dense, saturated regime.

The treatment of the prompt photon production can be developed within the QCD color dipole (CD) formalism, where the production mechanism resembles a bremsstrahlung $[13,14]$. The photon emission is viewed as a quark (or antiquark) electromagnetic bremsstrahlung, which exchange a single gluon with the target [15]. Hence, one can interpret the real photon radiation process in terms of $q \bar{q}$ dipole 
scattering off the target. The main ingredient in the CD approach is the universal dipole cross section, fit to DIS data, and that successfully describes the Hadron-Electron Ring Accelerator (HERA) at DESY (DESY-HERA) ep data for inclusive and exclusive processes. The dipole cross section takes into account the nonlinear gluon recombination effect that is expected to be relevant at low $x$. In the parton saturation picture, a scaling property associated with the DIS takes place, namely, a geometric scaling phenomenon. The cross sections for photon-target processes are a function of a single dimensionless scaling variable [16], instead of two independent variables, such as $x$ and $Q^{2}$ (photon virtuality). Such a property can be extended to single-particle production in hadron-hadron or $p(d) A$ collisions. We will show that it can explain the $x_{T}$ scaling observed in prompt photon production in $p p, d A$, and $p A$ reactions at central rapidities.

In this work, predictions are done for the nuclear modification factor considering RHIC and LHC kinematic regimes. Direct photon production at large- and low- $p_{T}$ in a wide rapidity range is considered. These results are an extension of the previous investigations presented in Ref. [17], where the differential cross section in $p p$ and $p A$ collisions at the LHC energies has been analyzed. Moreover, we carefully examine the theoretical mechanism responsible for the observed $x_{T}$ scaling in $p p$ and/or $p A$ collisions.

The paper is organized as follows. In Sec. II the theoretical framework is presented, including the main expressions used in our calculations within the CD formalism. In Sec. III we show our theoretical results, discussing and comparing them with the measurements available at RHIC and the LHC. The last section presents the main conclusions and remarks.

\section{THEORETICAL FORMALISM}

The nuclear modification factor $R_{p A}$ is determined as the ratio of $p A$ to $p p$ cross sections properly scaled with the correspondent mass number $A$ of the target nucleus,

$$
R_{p A}^{\gamma}\left(y, p_{T}\right)=\frac{d^{3} \sigma(p A \rightarrow \gamma A) / d y^{\gamma} d^{2} \overrightarrow{p_{T}}}{A \times d^{3} \sigma(p p \rightarrow \gamma p) / d y^{\gamma} d^{2} \overrightarrow{p_{T}}} .
$$

The advantage in using $R_{p A}$ consists in the cancellation of uncertainties that came from the individual cross sections in the ratio. The differential cross section for prompt photon production in $p p$ collisions in terms of the photon rapidity $y^{\gamma}$ and transverse momentum $p_{T}$ is obtained by convoluting the proton structure function with the partonic cross section derived in the Appendix B of Ref. [18] applied for direct photon production:

$$
\begin{gathered}
\frac{d^{3} \sigma(p p \rightarrow \gamma X)}{d y^{\gamma} d^{2} \vec{p}_{T}}=\frac{\alpha_{e m}}{2 \pi^{2}} \int_{x_{1}}^{1} \frac{d \alpha}{\alpha} F_{2}^{(P)}\left(\frac{x_{1}}{\alpha}, \mu^{2}\right) \frac{d^{3} \sigma(q p \rightarrow \gamma X)}{d y^{\gamma} d^{2} \vec{p}_{T}} \\
=\frac{\alpha_{e m}}{2 \pi^{2}} \int_{x_{1}}^{1} \frac{d \alpha}{\alpha} F_{2}^{(P)}\left(\frac{x_{1}}{\alpha}, \mu^{2}\right)\left\{m_{q}^{2} \alpha^{4}\left[\frac{\mathcal{I}_{1}}{\left(p_{T}^{2}+\varepsilon^{2}\right)}-\frac{\mathcal{I}_{2}}{4 \varepsilon}\right]\right. \\
\left.+\left[1+(1-\alpha)^{2}\right]\left[\frac{\varepsilon p_{T} \mathcal{I}_{3}}{\left(p_{T}^{2}+\varepsilon^{2}\right)}-\frac{\mathcal{I}_{1}}{2}+\frac{\varepsilon \mathcal{I}_{2}}{4}\right]\right\},
\end{gathered}
$$

where $F_{2}^{(P)}$ stands for the structure function for the projectile $(P)$ particle and $\mathcal{I}_{1}, \mathcal{I}_{2}$, and $\mathcal{I}_{3}$ are Hankel integral transforms of order $0\left(\mathcal{I}_{1}\right.$ and $\left.\mathcal{I}_{2}\right)$ and order $1\left(\mathcal{I}_{3}\right)$ given by

$$
\begin{aligned}
& \mathcal{I}_{1}=\int_{0}^{\infty} d r r J_{0}\left(p_{T} r\right) K_{0}(\varepsilon r) \sigma_{\mathrm{dip}}\left(x_{2}, \alpha r\right), \\
& \mathcal{I}_{2}=\int_{0}^{\infty} d r r^{2} J_{0}\left(p_{T} r\right) K_{1}(\varepsilon r) \sigma_{\mathrm{dip}}\left(x_{2}, \alpha r\right), \\
& \mathcal{I}_{3}=\int_{0}^{\infty} d r r J_{1}\left(p_{T} r\right) K_{1}(\varepsilon r) \sigma_{\mathrm{dip}}\left(x_{2}, \alpha r\right) .
\end{aligned}
$$

In numerical calculations we will consider a $F_{2}^{(P)}$ parametrization given in Ref. [19] (for proton and deuterium) and $\mu^{2}=$ $p_{T}^{2}$. The choice of the scale $\mu^{2}$ is one of the theoretical uncertainties in the formalism. Moreover, the fraction of the quark momentum carried by the photon is denoted by $\alpha$ and momentum fractions $x_{1}$ and $x_{2}$ have the form $x_{1}=\frac{p_{T}}{\sqrt{s}} e^{+y^{\gamma}}$ and $x_{2}=\frac{p_{T}}{\sqrt{s}} e^{-y^{\gamma}}$, where $\sqrt{s}$ is the collision center-of-mass energy. In the Hankel transforms, an effective quark mass appears in the auxiliary variable $\epsilon^{2}=\alpha^{2} m_{q}^{2}$, which is taken as $m_{q}=0.2$ $\mathrm{GeV}$ in our calculations.

Another quantity that enters the Hankel transforms is the dipole cross section $\sigma_{\text {dip }}$, a crucial ingredient to perform a calculation that can be compared with experimental measurements. Common features presented by $\sigma_{\text {dip }}$ are (i) it saturates for large dipole transverse sizes $r$, i.e., $\sigma_{\text {dip }} \rightarrow \sigma_{0}$; (ii) for small dipole sizes the dipole cross section behaves like $\sigma_{\text {dip }} \sim r^{2}$, i.e., vanishes accordingly with the color transparency phenomenon [20]. Here, we use models for dipole cross sections based on the idea of gluon saturation and constrained by recent data available from $e p$ collisions at the DESY-HERA collider. Explicitly, the following parametrizations will be considered: the Golec Biernat-Wüsthoff (GBW) model [21], with more recent fitting parameters reported in Ref. [22], and the Impact Parameter Saturation (IPSAT) model [23], where the parameters are given in Ref. [24]; such an approach includes QCD gluon evolution via the DokshitzerGribov-Lipatov-Altarelli-Parisi (DGLAP) equation. It should be noticed that, in the color transparency regime, the Hankel integrals can be analytically performed [25]. We will discuss this case in detail when the $x_{T}$ scaling is studied.

For a heavy target, nuclear effects are related to multiple parton scattering as well as to nonlinear gluon recombination. We employ state-of-art of phenomenological models to the dipole-nucleus amplitudes $N_{A}$, which contain explicit impact-parameter dependence or geometric scaling. There are basically two ways to implement the nuclear effects within the CD approach: (i) geometric scaling (GS) property from parton saturation models; and (ii) the Glauber-Gribov (GG) formalism for nuclear shadowing. First, we follow Ref. [26] to apply GS, including the $A$ dependence, in the scattering cross section. There, the authors demonstrated that the nuclear DIS cross section at small $x$ is directly associated with the cross section for DIS off a proton target. Hence, the proposed GS assumes that the nuclear effects are absorbed into the saturation scale and on the nucleus transverse area, $S_{A}=\pi R_{A}^{2}$, compared with the proton case, $S_{p}=\pi R_{p}^{2}$. Consequently, the saturation 
scale in protons, $Q_{s, p}$, is replaced by a nuclear saturation scale, $Q_{s, A}$, which is translated into an $A$ dependence,

$$
\begin{gathered}
Q_{s, A}^{2}=Q_{s, p}^{2}\left(\frac{A \pi R_{p}^{2}}{\pi R_{A}^{2}}\right)^{\Delta}, \\
N_{A}(x, r, b)=N\left(r Q_{s, p} \rightarrow r Q_{s, A}\right),
\end{gathered}
$$

which grows with the quotient $\Delta=1+\xi$ with $\xi=[(1-$ $\delta) / \delta$ ]. Here, $R_{A} \simeq 1.12 A^{1 / 3} \mathrm{fm}$ is the nucleus radius, whereas the quantities $\delta=0.79$ and $\pi R_{p}^{2}=1.55 \mathrm{fm}^{2}$ have been determined by data [26]. The prompt photon production cross section in $p A$ is rescaled accordingly as follows,

$$
\frac{d^{3} \sigma(p A \rightarrow \gamma X)}{d y d^{2} \vec{p}_{T}}=\left.\left(\frac{S_{A}}{S_{p}}\right) \frac{d^{3} \sigma(p p \rightarrow \gamma X)}{d y d^{2} \vec{p}_{T}}\right|_{Q_{s, p}^{2} \rightarrow Q_{s, A}^{2}} .
$$

We mention Ref. [27] where the GS property has been employed in order to describe data for both the DVCS at DESY-HERA and the exclusive meson production at the DESY-HERA and LHC colliders.

Otherwise, in terms of the GG formalism, which includes the multiple elastic-scattering diagrams related to the dipolenucleus interaction, the nuclear scattering cross section is written as [28]

$$
\sigma_{\text {dip }}^{\text {nuc }}(x, \vec{r} ; A)=2 \int d^{2} b\left\{1-\exp \left(-\frac{1}{2} \sigma_{\text {dip }}(x, r) T_{A}(b)\right)\right\},
$$

with $\sigma_{\text {dip }}$ being the dipole-proton cross section and $T_{A}$ being the nuclear profile function obtained from the Woods-Saxon distribution. Such a model was considered in Ref. [28], showing results in good agreement with the existing experimental data on the ratios of nuclear structure functions, $F_{2}^{A} / F_{2}^{B}$.

Still on the color transparency regime within the $\mathrm{CD}$ picture, a scaling property for the invariant cross section of prompt photon in $p p$ and/or $p A$ collisions on the variable $x_{T}=2 p_{T} / \sqrt{s}$ (the so-called $x_{T}$ scaling) can be derived. Taking the massless limit, $m_{q} \rightarrow 0$ in Eq. (3), the second term holds, with the only contribution that survives from the Hankel integrals being proportional to an analytic function, $\mathcal{I}_{1} \propto$ $\sigma_{0}\left(\alpha Q_{s}\right)^{2} / p_{T}^{4}$. This last result is a consequence of considering the color transparency in the dipole-target cross section. Furthermore, a rough approximation can be obtained for the nucleon structure function assuming the GBW model (with $\left.\gamma_{s}=1\right)$,

$$
F_{2}\left(x, Q^{2}\right) \approx \frac{\sigma_{0} Q^{2}}{4 \pi^{2} \alpha_{e m}}\left(\frac{Q_{s}^{2}(x)}{Q^{2}}\right)^{\gamma_{s}},
$$

where the saturation scale is set as $Q_{s}^{2}(x)=Q_{0}^{2}\left(x_{0} / x\right)^{\lambda}$ (with $Q_{0}=1 \mathrm{GeV}$ and parameters $x_{0}$ and $\lambda$ being fit from HERA data at small $x$ ). Hence, taking into account the assumptions established above and further integrating Eq. (3) over $\alpha$, an $x_{T}$-scaling expression is obtained for the $p p$ case,

$$
E \frac{d^{3} \sigma^{p p \rightarrow \gamma X}}{d^{3} p}\left(x_{T}\right) \approx \frac{N_{0}}{(\sqrt{s})^{4}}\left(\frac{x_{T}}{2}\right)^{-n} f\left(x_{1}\right),
$$

with $\quad n=2 \lambda+4 \simeq 4.5 \quad$ and $\quad f\left(x_{1}\right) \approx(1012 / 1989)-$ $(4 / 17) x_{1}^{17 / 4}+(8 / 13) x_{1}^{13 / 4}-(8 / 9) x_{1}^{9 / 4}$ being a well- behaved function of $x_{1}=\left(x_{T} / 2\right) e^{y}$ resulting from the $\alpha$ integration. Moreover, the overall normalization is given by $N_{0}=\bar{\sigma}_{p p}\left(x_{0}\right)^{2 \lambda}$, with parameters $\bar{\sigma}_{p p}=0.035 \mathrm{mb} / \mathrm{GeV}^{2}$, $x_{0}=0.4 \times 10^{-4}$, and $\lambda=0.248$ taken from the GBW model. On the other hand, for $p A$ collisions based on GS proposed in Ref. [26], the invariant cross section reads

$$
\begin{aligned}
E \frac{d^{3} \sigma}{d^{3} p}(p A \rightarrow & \gamma X) \approx \frac{N_{0}}{(\sqrt{s})^{4}}\left(\frac{S_{A}}{S_{p}}\right)\left(\frac{A S_{p}}{S_{A}}\right)^{\Delta}\left(\frac{x_{T}}{2}\right)^{-n} f\left(x_{1}\right) \\
& \approx A\left(\frac{A S_{p}}{S_{A}}\right)^{\xi} E{\frac{d^{3} \sigma}{d^{3} p}}^{p p \rightarrow \gamma X}\left(x_{T}\right)
\end{aligned}
$$

where $\xi=(1-\delta) / \delta \simeq 0.27$. The value of $\bar{\sigma}_{p p}$ is determined in order to describe the lower-energy data in $p p$ collisions, and we set the same value for $p A$ reactions. The simple parametrization presented above can be further sophisticated by leaving the anomalous dimension $\gamma_{s}$ as a free parameter or using a $p_{T}$ dependence like in the Boer-Utermann-Wessels (BUW) model [29]. Similar proposals of scaling can be found in Refs. [30-32], where the scaling observed in prompt photon production is related to an universal multiplicity scaling. The latter is studied by using the charged hadron pseudorapidity density at midrapidity, $d N_{h} / d \eta$.

In the next section a comparison is made between the theoretical approach based on QCD dipole picture and experimental data from the RHIC and LHC colliders.

\section{RESULTS AND DISCUSSIONS}

In this section, we present the numerical calculations concerning the nuclear modification factor $R_{p A}$ obtained with the CD approach, where we used the GBW and IPSAT phenomenological models for the dipole cross section. We investigate the influence of nuclear effects in low- and large- $p_{T}$ prompt photon production via GS and GG formalism. Some comments are in order here. We compute the dipole-nucleus amplitude considering the GBW model as an input for GS and GG implementations. In our calculations with the IPSAT model we are applying the small- $r$ limit for $\sigma_{\text {dip }}$, which is appropriate in the large- $p_{T}$ domain given that $r \approx 1 / p_{T}$ in direct photon production and also enables us to analytically solve the Hankel transforms discussed in the previous section. Furthermore, there is no significant change regarding the option for the proton structure function in Eq. (3). It has been verified that employing $F_{2}^{p}$ from Ref. [19] or the ALLM2007 parametrization [33] results in nearly identical numerical results. As a last remark, the CD approach has a threshold of validity taken as $x_{2} \leqslant 10^{-2}$, which is, in principle, well suitable for small $x_{2}$. However, Ref. [25] demonstrates that a large- $x$ correction should be added to consistently describe the prompt photon phenomenology. Therefore, we have multiplied the GBW dipole cross section by a threshold factor $\left(1-x_{2}\right)^{n}$ (with $n=7$ ). In the IPSAT model, the parametrization for the gluon PDF already contains the threshold factor.

Now, in Fig. 1 we show the results for the nuclear modification factor in $p \mathrm{~Pb}$ collisions at $\sqrt{s}=8.16 \mathrm{TeV}$ compared with the measurements by the ATLAS experiment [34] as a 

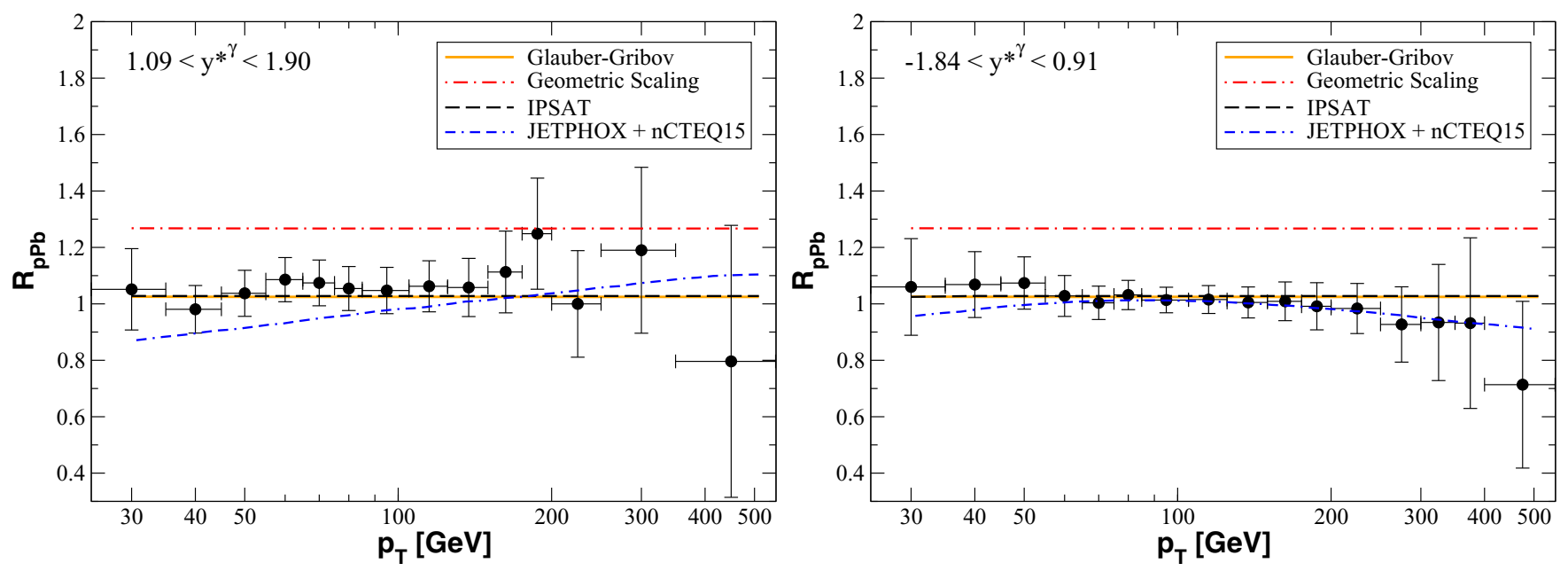

FIG. 1. Nuclear modification factor $R_{p \mathrm{~Pb}}$ as a function of $p_{T}$ shown for a forward and backward rapidity bins with respect to the centerof-mass rapidity $y^{* \gamma}$ at $\sqrt{s}=8.16 \mathrm{TeV}$. The predictions are obtained using GG, GS, and IPSAT models. Results from JETPHOX Monte Carlo using the nPDF nCTEQ15 are also presented as a matter of comparison, together with experimental data from the ATLAS Collaboration [34].

function of $p_{T}$ and $y^{* \gamma}$, the latter being the center-of-mass rapidity. Moreover, the results with $\mathrm{pQCD}$ at next-to-leading order level of direct and fragmentation contributions to the cross sections using the JETPHOX Monte Carlo [35] and nCTEQ15 nuclear PDF [36] are also included in order to perform a comparison with our results. Considering the two rapidity bins, the measured nuclear modification factor is consistent with unity, indicating that the magnitude of nuclear effects becomes negligible. In addition, the GG and IPSAT approaches predict a quite small nuclear effect, while the GS model predicts $R_{p A} \gtrsim 1$. Concerning the GS approach, the nuclear ratio has the form [see Eqs. (12) and (14)]

$$
R_{p A}^{\gamma} \approx\left(\frac{A \pi R_{p}^{2}}{\pi R_{A}^{2}}\right)^{\xi}
$$

with $\xi \simeq 0.27$. Accordingly, this reproduces numerically $R_{p \mathrm{~Pb}} \simeq 1.3$ for any value of $p_{T}$. For the IPSAT case, the small- $r$ approximation allows us to expand the eikonalized amplitudes as $N_{p} \approx\left(\pi^{2} \alpha_{s} / 2 N_{c}\right) r^{2} x g T_{p}(b)$ and $N_{A} \approx$ $\left(\pi^{2} \alpha_{s} / 2 N_{c}\right) r^{2} x g T_{A}(b)$. Besides, the normalization of the proton and nuclear thickness function, $\int d^{2} \vec{b} T_{A}(b)=A(A=1$ for the proton case), implies that $\sigma_{\text {dip }}^{\text {nuc }}=A \sigma_{\text {dip }}$ and results in $R_{p \mathrm{~Pb}} \approx 1$. The GG and IPSAT results are fairly similar to those from JETPHOX Monte Carlo with nCTEQ15. We will see that the situation changes in the low- $p_{T}$ case.

To test the nuclear effects that have been addressed, our predictions for $R_{p A}$ are compared with recent studies in the literature employing others approaches. We start discussing Ref. [37], where calculations are performed considering $p \mathrm{~Pb}$ collisions at an energy of $8 \mathrm{TeV}$ and based on CGC formalism using $\mathrm{CD}$ cross sections solved from the running coupling Balitsky-Kovchegov (BK) evolution equation (see, e.g., Refs. [38,39] where the CGC formalism is employed for the investigation of direct photons in $e$ A collisions). The CGC formalism predicts a consistent suppression at forward rapidities in the range $1 \leqslant p_{T} \leqslant 8 \mathrm{GeV}$. In Fig. 2 we present the $R_{p A}^{\gamma}$ predictions at low- $p_{T}$ for fixed values of the photon forward rapidity, $y^{\gamma}=3,4$, and 5 , respectively. Up to $p_{T} \approx 2$ $\mathrm{GeV}, \mathrm{GG}, \mathrm{GS}$, and CGC models predict similar results with a suppression pattern. This is well understood in terms of the nuclear saturation scale. At low transverse momentum and forward rapidities, small $x_{2}=\left(x_{T} / 2\right) e^{-y}$ is probed. For instance, at $p_{T}=2 \mathrm{GeV}$ and $y^{\gamma}=4$, one has $x_{2} \approx 6.6 \times 10^{-6}$ and the proton saturation scale reaches $Q_{s, p}^{2} \simeq 1.7 \mathrm{GeV}^{2}$ and the corresponding nuclear saturation scale $Q_{s, \mathrm{~Pb}}^{2} \approx 3 Q_{s, p}^{2} \simeq$ $5 \mathrm{GeV}^{2}$. As we can see, one has $p_{T}^{2} \leqslant Q_{s, A}^{2}$ at low $p_{T}$ and at forward rapidities at the $\mathrm{LHC}$, expecting an important shadowing correction. As $p_{T}$ increases at fixed rapidity, a transition from saturated to dilute regime is reached, $p_{T}^{2} \gg Q_{s, A}^{2}$, and nuclear corrections are weaker. An enhancement of $R_{p \mathrm{~Pb}}$ is verified as excepted for the IPSAT model. However, the results with GG and CGC tend to be closer to unity at $p_{T} \geqslant 8$ $\mathrm{GeV}$, in contrast with GS that continues showing an enhancement of $R_{p \mathrm{~Pb}}$. A Cronin enhancement has been observed in both GG and GS results. Moreover, the location of the peak depends on the rapidity and it moves in the direction of larger $p_{T}$ in accordance with the increase in rapidity. The peaks have the same shape in both predictions and differ in their height.

The Cronin-like peak is typical in models including rescattering, mostly at midrapidities. In Ref. [7], this issue was first investigated and it was found that the Cronin enhancement can survive at the LHC energy within the saturation QCD dipole models. We observe the same pattern for GG and GS calculations. This is consistent with recent studies on pion-photon correlations presented in Ref. [5]. In Ref. [7], the peak height can be reduced if gluon shadowing, $R_{G}$ (from $|q \bar{q} g\rangle$ Fock state contribution), in the form $\sigma_{\mathrm{dip}} \rightarrow R_{G}\left(x_{2}\right) \sigma_{\mathrm{dip}}$, enters in Eq. (10). Namely, a subtle cancellation between the saturation and gluon shadowing effects can lead to a rather small Cronin peak in $R_{p A}^{\gamma}$. It is clear that further experimental analyses of the ratio $R_{p A}^{\gamma}$ at very forward $y^{\gamma}$ would be very fruitful to draw a distinction between the CGC and its competing approaches.

The discussion above can be placed in juxtaposition with results from the usual pQCD approach. At leading order (LO) 

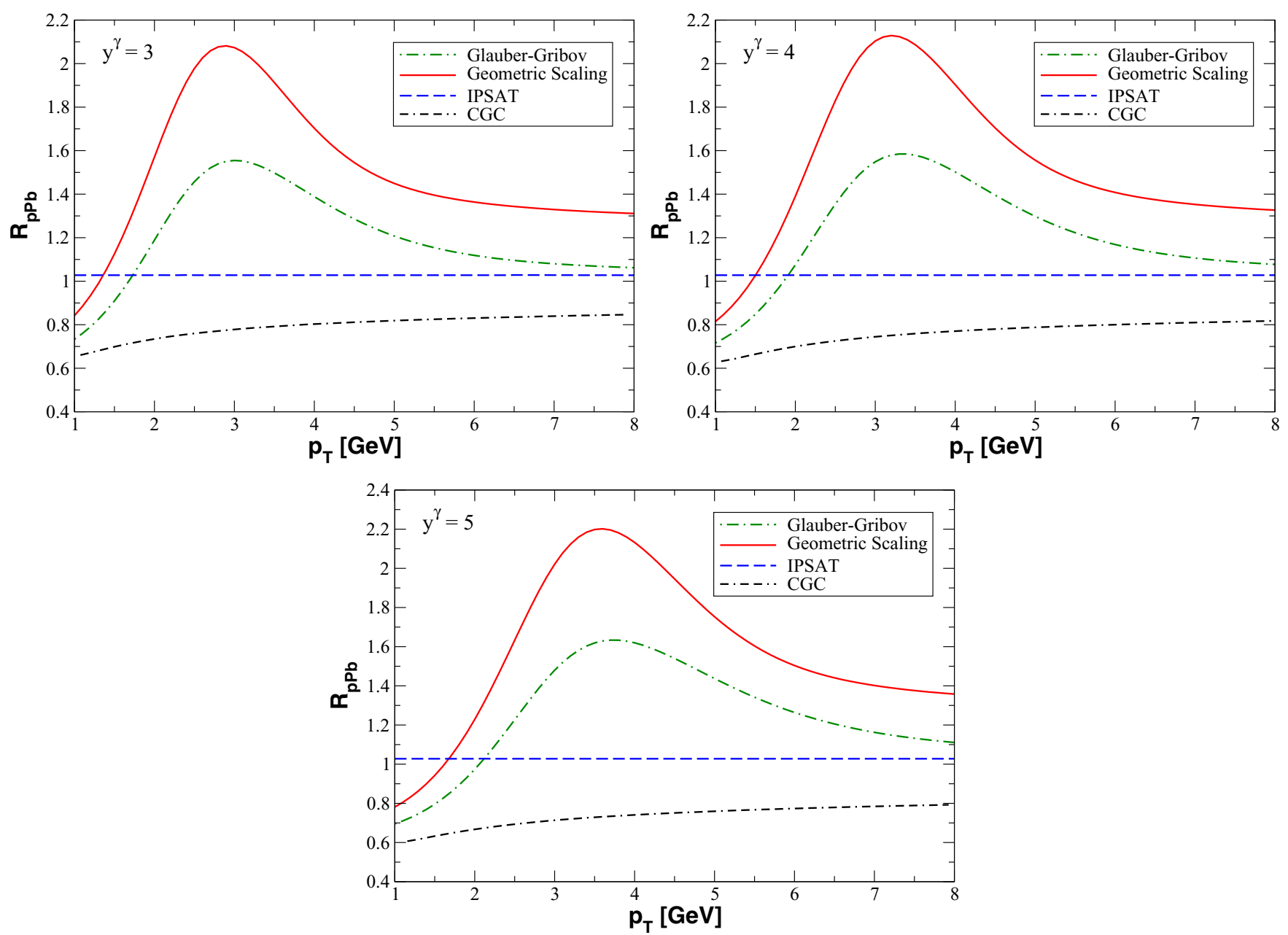

FIG. 2. Nuclear modification factor $R_{p \mathrm{~Pb}}$ for prompt photon as a function of $p_{T}$ shown for different fixed values of the photon forward rapidity at $\sqrt{s}=8 \mathrm{TeV}$. The predictions are obtained using GG, GS, and IPSAT approaches and compared with the results from CGC effectivefield theory.

accuracy, it can be shown [40] for central rapidities, $y^{\gamma} \approx 0$, that $R_{p A}^{\gamma}\left(x_{T}\right) \simeq \frac{1}{2}\left[R_{F_{2}}^{A}\left(x_{T}\right)+R_{G}^{A}\left(x_{T}\right)\right]$. That is to say, the nuclear modification factor is a linear combination of nuclear ratio for gluons, $R_{G}$, and nuclear ratio for structure functions in a nucleus $A$, and at midrapidity both contributions have similar weights. On the other hand, at forward rapidities, $y^{\gamma}>y_{c}^{\gamma}$ (let us say $y_{c}^{\gamma} \approx 2$, where $y_{c}^{\gamma}$ refers to a value taken as a large rapidity), the relation becomes $R_{p A}^{\gamma}\left(x_{T}, y^{\gamma}\right) \simeq R_{G}^{A}\left(x_{T} e^{-y^{\gamma}}\right)$ and the pQCD approach is quite close to ours. The relations between the nuclear modification factors and the nuclear gluon PDF and nuclear structure functions, $F_{2}^{A}$ were shown to remain as a correct approximation up to a few-percent accuracy in calculations at the next-to-leading order (NLO) level [40]. We quote Refs. [12,41-43], which compare different nuclear PDFs and study theoretical uncertainties that are due to their uncertainties and scale variations. As a remark on the experimental side, studies of direct photons at the energy $\sqrt{s}=8.16$ $\mathrm{TeV}$ at $\mathrm{LHCb}$ [44] for $p$ - $\mathrm{Pb}$ and $\mathrm{Pb}-p$ are now well underway. They probe small- $p_{T}$ regions at very forward rapidities, which is ideal for investigating the nuclear effects in the gluon sector.
Additionally, for the purpose of continuing to make a comparison with predictions from the CGC framework [37], we show in Fig. 3 the results for the nuclear modification factor in $p \mathrm{Au}$ collisions at RHIC at $\sqrt{s}=200 \mathrm{GeV}$ and two-photon forward rapidity bins $2.5<y^{\gamma}<3.2$ and $3.2<y^{\gamma}<4$. Here, we found the same behavior pattern regarding the results as seen in the $p \mathrm{~Pb}$ case at $\sqrt{s}=8 \mathrm{TeV}$. Namely, $R_{p \mathrm{Au}}<2$ at small values of $p_{T}$ and points out that the nuclear effects are not perceptible towards larger $p_{T}$. However, the approaches predict less suppression at small $p_{T}$ at RHIC energy and reach unity faster in comparison to the previous case. Interestingly, the GG and the CGC approach give similar results at sufficiently high $p_{T}$ in the two rapidity bins. The Cronin peak remains at low $p_{T}$ for $\mathrm{GG}$ and $\mathrm{GS}$, similar to $p A$ collisions at the LHC.

For the sake of completeness, we present in Fig. 4 the predictions considering the energy of $\sqrt{s}=200 \mathrm{GeV}$ at RHIC for minimum-bias $d \mathrm{Au}$ collisions at midrapidity. We include the experimental data for $R_{d \mathrm{Au}}$ extracted by the PHENIX Collaboration [45] for $p_{T}>4 \mathrm{GeV}$, skipping the data for $p_{T}<4$ $\mathrm{GeV}$ which refers to the virtual photon measurement and is 

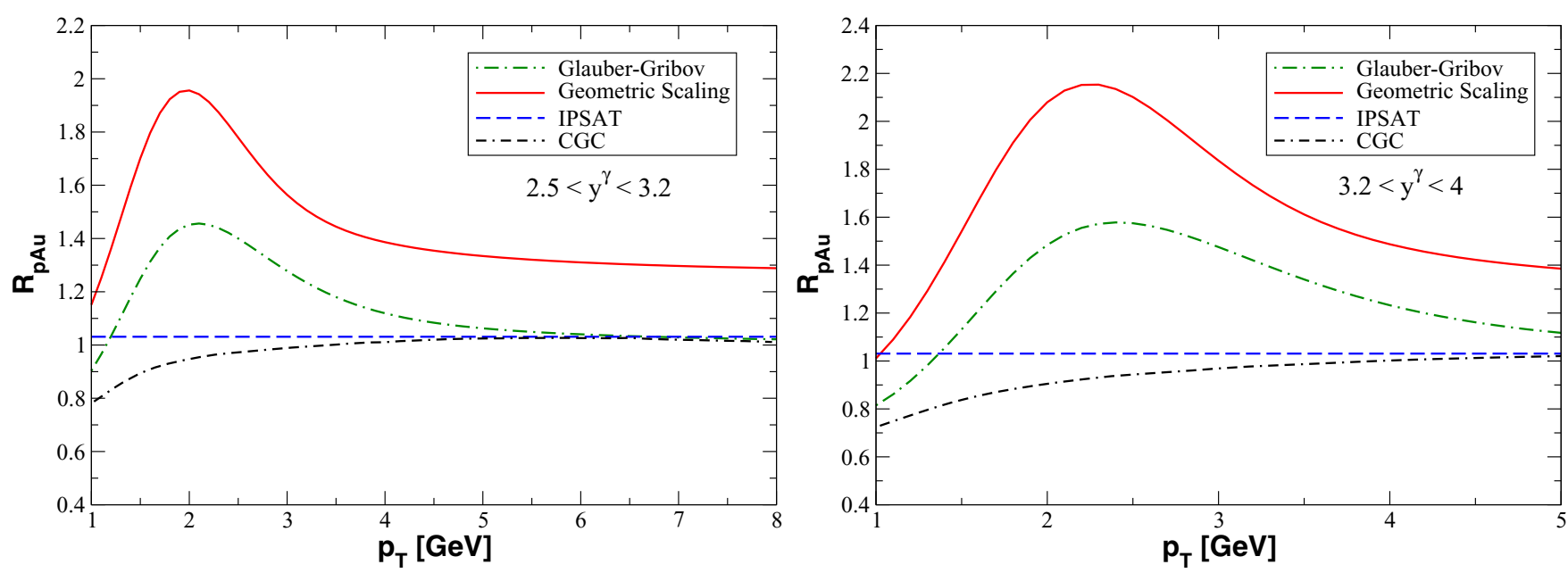

FIG. 3. Nuclear modification factor $R_{p \text { Au }}$ for prompt photon as a function of $p_{T}$ shown for two-photon forward rapidity bins at $\sqrt{s}=200$ $\mathrm{GeV}$. The predictions are obtained using GG, GS, and IPSAT approaches and compared with the results from CGC effective-field theory.

outside the scope of this paper. The ratio is defined as

$$
R_{d \mathrm{Au}}^{\gamma}=\frac{d N^{d+\mathrm{Au} \rightarrow \gamma+X} / d y d^{2} \overrightarrow{p_{T}}}{\left\langle N_{\mathrm{coll}}\right\rangle d N^{p+p \rightarrow \gamma+X} / d y d^{2} \overrightarrow{p_{T}}},
$$

where $\left\langle N_{\text {coll }}\right\rangle$ is the average number of $N N$ binary collisions.

Predictions are compared with the calculations from Ref. [46] (dot-dashed line) with different combinations of initial-state effects (Cronin enhancement, isospin correction, nuclear effects embedded in nPDFs, and initial-state parton energy loss), i.e., cold nuclear matter (CNM) effects. The GG and IPSAT results are not dependent on $p_{T}$, producing a constant ratio of order 0.9 and quite similar to the CNM results. The GS prediction presents the same pattern as at LHC energies and central rapidities, with $R_{p A}^{\gamma} \sim 1.3$. The main uncertainty in the GS approach is the prescription for the

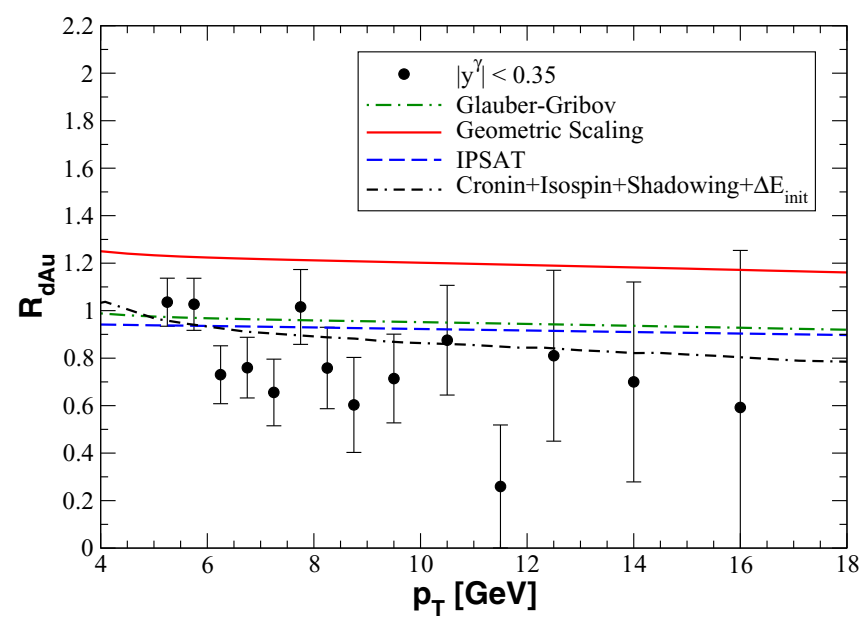

FIG. 4. Nuclear modification factor $R_{d \mathrm{Au}}$ as a function of $p_{T}$ in midrapidity at $\sqrt{s}=200 \mathrm{GeV}$. The predictions are obtained using GG, GS, and IPSAT approaches and compared with the pQCD calculations including CNM effects and with the experimental data from the PHENIX Collaboration [45]. nuclear saturation scale, $Q_{s, A}^{2}$, and in Ref. [17] we determined the uncertainty being $\approx 20 \%$.

Finally, we present the results concerning the $x_{T}$ scaling observed in prompt photon production at central rapidities, $y^{\gamma} \approx 0$. Hence, the invariant cross section for inclusive particle production can be expressed as

$$
E \frac{d^{3} \sigma}{d^{3} p}=\frac{G\left(x_{T}\right)}{[\sqrt{s}]^{n_{\mathrm{eff}}\left(\sqrt{s}, x_{T}\right)}} .
$$

The scaling works for almost all the available data, with the power of the invariant cross section becoming softer towards higher $x_{T}$. The effective power is empirically determined as $n_{\text {eff }}=4.5$ [47-49]. The usual pQCD approach without hard scale evolution predicts the invariant cross section being proportional to $[\sqrt{s}]^{4}[47]$.

For illustration, the scaled cross sections are presented for $p p$ [50,51] and $d \mathrm{Au}$ [45] collisions at RHIC and $p p / p \mathrm{~Pb}$ collisions at LHC. Figure 5 shows our predictions for the invariant cross sections in terms of $x_{T}=2 p_{T} / \sqrt{s}$ compared with the data collected for $y^{\gamma} \approx 0$ by PHENIX at $\sqrt{s}=200$ $\mathrm{GeV}$, ATLAS at $\sqrt{s}=8$ and $13 \mathrm{TeV}[52,53]$ as well as the CMS data at $13 \mathrm{TeV}$ [54]. In the $p p$ case (left panel), the analytic scaling curve, Eq. (12), is shown for the limiting energies of $\sqrt{s}=200 \mathrm{GeV}$ (dot-dashed line) and $\sqrt{s}=13 \mathrm{TeV}$ (solid line). The scaling curves have the correct shape at small $x_{T}$, however the correct transition to large $x_{T}$ is not achieved. As discussed before, this would be solved if the quantity $\gamma_{s}$ becomes $p_{T}$ dependent (as in the BUW dipole model). It is remarkable the good agreement with a full calculation using the IPSAT model and the experimental measurements for any $p_{T}$. It is presented for $\sqrt{s}=8 \mathrm{TeV}$ (long-dashed line) and we verified low sensitivity to the energy value.

The same procedure is followed in the proton(deuterium)nucleus case. We have normalized the invariant cross sections by $A B$, i.e., we considered the spectra per nucleon. We explicitly present the results for the analytical expression in Eq. (14) in the limiting energies of $\sqrt{s}=200 \mathrm{GeV}$ (dot-dashed line) and $\sqrt{s}=8.16 \mathrm{TeV}$ (solid line). The IPSAT result for $\sqrt{s}=$ 

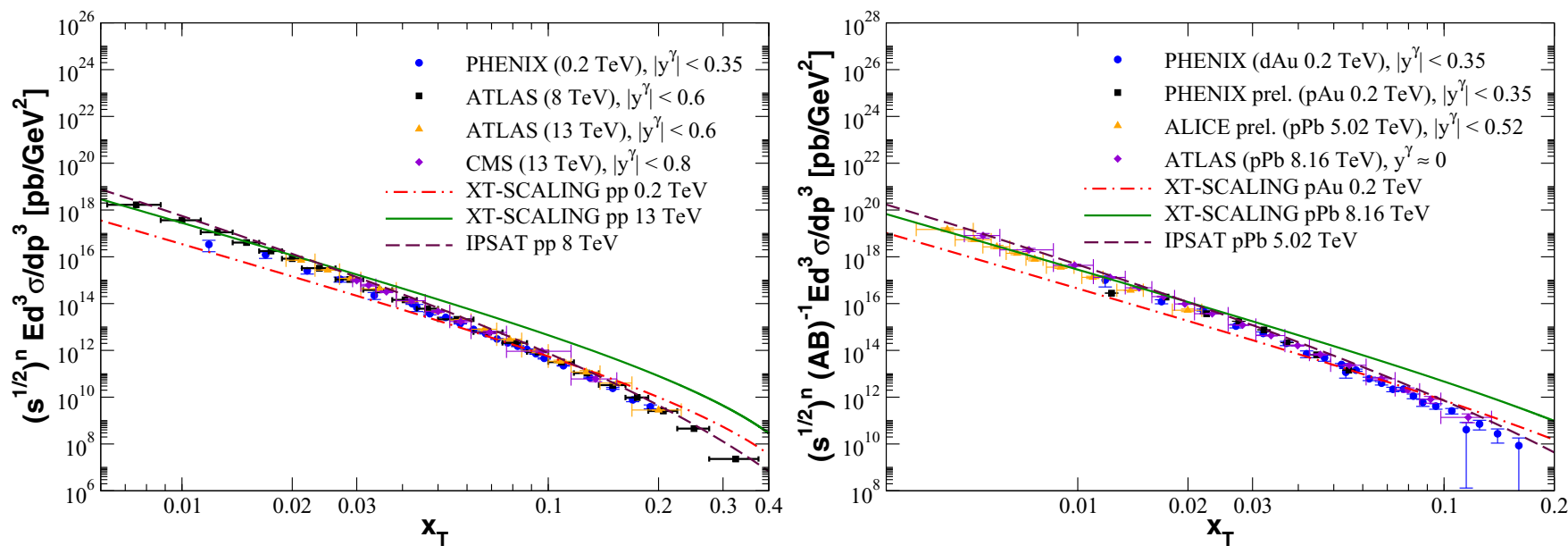

FIG. 5. The $x_{T}$ scaling of prompt photon production in $p p$ (left panel) and $d \mathrm{Au} / p \mathrm{Au} / p \mathrm{~Pb}$ (right panel, normalized by nucleons number) collisions at midrapidity. Analytic expressions of Eqs. (12) and (14) are presented in the limiting energies. The full calculation using the IPSAT model (including DGLAP evolution, color transparency approximation) is shown at fixed energies of $\sqrt{s}=8 \mathrm{TeV}(p p)$ and $\sqrt{s}=5.02 \mathrm{TeV}$ $(p A)$.

$5.02 \mathrm{TeV}$ is represented by the long-dashed curve. There is a clear resemblance between the two $p p$ and $p(d) A$ cases. The published data from $(d \mathrm{Au})$ PHENIX $[45]$ and $(p \mathrm{~Pb})$ ATLAS [34] are included. For the sake of illustration, we also included the preliminary data from PHENIX for $p$ Au collisions $(\sqrt{s}=$ $200 \mathrm{GeV})[55,56]$ as well as the preliminary ALICE $p \mathrm{~Pb}$ data $(\sqrt{s}=5.02 \mathrm{TeV})[57]$.

Interestingly, PHENIX Collaboration [56] has recently investigated the scaling of the direct photon yield, integrated for $p_{T} \geqslant 1.0 \mathrm{GeV}$, as a function of charged-particle multiplicity, $d N_{c h} /\left.d \eta\right|_{\eta=0}$. A direct photon excess yield was demonstrated at small $p_{T}$ in central $p \mathrm{Au}$ collisions above $N_{\text {coll }}$ scaled baseline fit for proton-proton collisions. The collaboration claims it may originate from existing QGP droplets in small central systems, suggesting the presence of a transition point between small and large systems. It would be interesting to address this question with the $\mathrm{CD}$ picture presented here.

\section{SUMMARY}

In this work, we estimate the nuclear modification factor for prompt photon production at energies available at RHIC and the LHC, considering distinct rapidity bins. We analyze the influence of nuclear effects in the transverse momentum distribution of prompt photons, correspondingly introduced by Glauber-Gribov, geometric scaling, and IPSAT (color transparency approximation) models. Our results do not indicate a strong suppression due the saturation effects, and there are no free parameters in the calculations. The experi- mental measurements of $R_{p A}$ in both RHIC and LHC colliders are consistent with unity within the experimental uncertainties at different values of rapidity. The models are in agreement with data in the $p_{T}$ region above $4 \mathrm{GeV}$. Besides, the GG and GS models predict a Cronin peak at the lower- $p_{T}$ region (below $4 \mathrm{GeV}$ ), which is expected as seen in the data from the PHENIX Collaboration in Ref. [45] for virtual photon measurements. Thus, we demonstrate that the CGC predictions are distinct from ours and this suggests that future experimental measurements on the nuclear modification factor at forward rapidities may be performed to discriminate the models.

Moreover, we have demonstrated that the parametrization proposed for the $x_{T}$ scaling of prompt photon production, considering proton and nuclear targets, works very well in describe the corresponding experimental measurements at low transverse momentum. This is notable given the simplicity of the parametrizations obtained within the QCD color dipole formalism in the massless-quark limit, which can be useful in data analysis of future experimental measurements of prompt photons.

\section{ACKNOWLEDGMENTS}

This work was partially financed by the Brazilian funding agencies CAPES, CNPq, and FAPERGS. This study was financed in part by the Coordenação de Aperfeiçoamento de Pessoal de Nível Superior - Brasil (CAPES) - Finance Code 001. G.G.S. acknowledges funding from the Brazilian agency Conselho Nacional de Desenvolvimento Científico e Tecnológico (CNPq) with grant CNPq/313342/2017-2.
[1] A. Accardi et al., Eur. Phys. J. A 52, 268 (2016).

[2] I. Arsene et al.(BRAHMS Collaboration), Phys. Rev. Lett. 93, 242303 (2004).

[3] J. Adams et al. (STAR Collaboration), Phys. Rev. Lett. 97, 152302 (2006).
[4] A. Adare et al. (PHENIX Collaboration), Phys. Rev. Lett. 107, 172301 (2011).

[5] V. P. Goncalves, Y. Lima, R. Pasechnik, and M. Sumbera, Phys. Rev. D 101, 094019 (2020) 
[6] J. Jalilian-Marian and A. H. Rezaeian, Phys. Rev. D 86, 034016 (2012).

[7] A. H. Rezaeian and A. Schafer, Phys. Rev. D 81, 114032 (2010).

[8] R. Pasechnik and M. Sumbera, Universe 3, 7 (2017).

[9] S. Acharya et al. (ALICE Collaboration), Phys. Rev. C 99, 024912 (2019).

[10] L. E. Gordon and W. Vogelsang, Phys. Rev. D 50, 1901 (1994).

[11] S. Frixione, Phys. Lett. B 429, 369 (1998).

[12] I. Helenius, K. J. Eskola, and H. Paukkunen, J. High Energy Phys. 09 (2014) 138.

[13] B. Z. Kopeliovich, A. Schafer, and A. V. Tarasov, Phys. Rev. C 59, 1609 (1999).

[14] B. Z. Kopeliovich, in Proceedings of the Workshop Hirschegg '95: Dynamical Properties of Hadrons in Nuclear Matter, Hirschegg, January 16-21, 1995, edited by H. Feldmeyer and W. Nörenberg (Darmstadt, 1995), p. 102.

[15] B. Z. Kopeliovich, A. H. Rezaeian, H. J. Priner, and I. Schmidt, Phys. Lett. B 653, 210 (2007).

[16] S. Munier and R. Peschanski, Phys. Rev. Lett. 91, 232001 (2003).

[17] G. Sampaio dos Santos, G. Gil da Silveira and M. V. T. Machado, Eur. Phys. J. C 80, 812 (2020).

[18] B. Z. Kopeliovich, J. Raufeisen, A. V. Tarasov, and M. B. Johnson, Phys. Rev. C 67, 014903 (2003).

[19] B. Adeva et al., Phys. Rev. D 58, 112001 (1998).

[20] Al. B. Zamolodchikov, B. Z. Kopeliovich, and L. I. Lapidus, JETP Lett. 33, 595 (1981).

[21] K. J. Golec-Biernat and M. Wusthoff, Phys. Rev. D 59, 014017 (1998).

[22] K. Golec-Biernat and S. Sapeta, J. High Energy Phys. 03 (2018) 102.

[23] H. Kowalski and D. Teaney, Phys. Rev. D 68, 114005 (2003).

[24] A. H. Rezaeian, M. Siddikov, M. Van de Klundert and R. Venugopalan, Phys. Rev. D 87, 034002 (2013).

[25] M. V. T. Machado and C. B. Mariotto, Eur. Phys. J. C 61, 871 (2009).

[26] N. Armesto, C. A. Salgado, and U. A. Wiedemann, Phys. Rev. Lett. 94, 022002 (2005).

[27] F. G. Ben, M. V. T. Machado, and W. K. Sauter, Phys. Rev. D 96, 054015 (2017).

[28] N. Armesto, Eur. Phys. J. C 26, 35 (2002).

[29] D. Boer, A. Utermann, and E. Wessels, Phys. Rev. D 77, 054014 (2008).
[30] C. Klein-Bósing and L. McLerran, Phys. Lett. B 734, 282 (2014).

[31] M. Praszałowicz, EPJ Web Conf. 206, 02002 (2019).

[32] V. Khachatryan and M. Praszałowicz, Eur. Phys. J. C 80, 670 (2020).

[33] D. Gabbert and L. De Nardo, arXiv:0708.3196.

[34] M. Aaboud et al. (ATLAS Collaboration), Phys. Lett. B 796, 230 (2019).

[35] P. Aurenche, J. P. Guillet, E. Pilon, M. Werlen, and M. Fontannaz, Phys. Rev. D 73, 094007 (2006).

[36] K. Kovarik et al., Phys. Rev. D 93, 085037 (2016).

[37] B. Ducloué, T. Lappi, and H. Mäntysaari, Phys. Rev. D 97, 054023 (2018).

[38] K. Roy and R. Venugopalan, J. High Energy Phys. 05 (2018) 013.

[39] I. Kolbé, K. Roy, F. Salazar, B. Schenke, and R. Venugopalan, arXiv:2008.04372.

[40] F. Arleo and T. Gousset, Phys. Lett. B 660, 181 (2008).

[41] M. Goharipour and H. Mehraban, Phys. Rev. D 95, 054002 (2017).

[42] M. Goharipour and S. Rostami, Phys. Rev. C 99, 055206 (2019).

[43] M. Klasen, C. Klein-Bösing, and H. Poppenborg, J. High Energy Phys. 03 (2018) 081.

[44] T. Boettcher (LHCb Collaboration), Nucl. Phys. A 982, 251 (2019).

[45] A. Adare et al. (PHENIX Collaboration), Phys. Rev. C 87, 054907 (2013).

[46] I. Vitev and B. W. Zhang, Phys. Lett. B 669, 337 (2008).

[47] W. Vogelsang and M. Whalley, J. Phys. G 23, A1 (1997).

[48] D. d'Enterria and J. Rojo, Nucl. Phys. B 860, 311 (2012).

[49] G. David, Rep. Prog. Phys. 83, 046301 (2020).

[50] S. S. Adler et al. (PHENIX Collaboration), Phys. Rev. Lett. 98, 012002 (2007).

[51] S. S. Adler et al. (PHENIX Collaboration), Phys. Rev. D 86, 072008 (2012).

[52] M. Aaboud et al. (ATLAS Collaboration), Phys. Lett. B 770, 473 (2017).

[53] G. Aad et al. (ATLAS Collaboration), J. High Energy Phys. 08 (2016) 005.

[54] A. M. Sirunyan et al. (CMS Collaboration), Eur. Phys. J. C 79, 20 (2019).

[55] V. Canoa Roman, MDPI Proc. 10, 32 (2019).

[56] V. Khachatryan (PHENIX Collaboration), Nucl. Phys. A 982 , 763 (2019).

[57] D. Blau (ALICE Collaboration), EPJ Web Conf. 222, 02001 (2019). 